\title{
Effect of Substitutional Point Defect of Gold (Au) in Indium (In) Site of Double Halide Perovskite $\left(\mathrm{Cs}_{2} \mathrm{InSbCl}_{6}\right)$
}

\author{
I. Magaji ${ }^{\mathrm{a}, *}$, A. Shuaibu ${ }^{\text {a }}$, M. S. Abubakar ${ }^{\mathrm{a}}$, M. Isah ${ }^{\mathrm{a}}$, A. Hussaini ${ }^{\mathrm{a}}$ \\ ${ }^{a}$ Department of Physics, Faculty of Science, Kaduna State University, P.M.B, 2339, Kaduna, Nigeria
}

\begin{abstract}
Lead $(\mathrm{Pb})$ free (non-toxic) perovskite solar cells materials have attracted great interest in the commercialization of the photovoltaic devices. In this work, density functional theory (DFT) and linear response time-dependent within density functional theory (TDDFT) are used to simulate and investigate the effect of gold ( $\mathrm{Au}$ ) doped $\mathrm{Pb}$-free double halide perovskite $\mathrm{A}_{2} \mathrm{BB} / \mathrm{X}_{6}(\mathrm{~A}=\mathrm{Cs} ; \mathrm{B}=\mathrm{In}, \mathrm{Au} ; \mathrm{B}$ ' $=\mathrm{Sb} ; \mathrm{X}=\mathrm{Cl})$ on the structural, electronic, and optical properties for perovskite solar cell application. On the structural properties, bond length and bulk modulus calculations show that the doped compound is more likely to resist deformation than the undoped compound. The calculated band structure for both materials (doped and undoped) reveals the presence of the Valence Band Maximum (VBM) and the Conduction Band Minimum (CBM) at around the same symmetry point which indicates a direct band gap nature (at $\Gamma$ - point). The band gap value for the initial compound ( $\mathrm{E}_{\mathrm{g}}=0.99 \mathrm{eV}$ ) agrees with published theoretical values. For the gold doped compound, the value of the band gap increased to a value of $1.25 \mathrm{eV}$. The result of the optical properties shows that the Au-doped material has higher absorption coefficient, lower reflectivity and higher optical conductivity when compared with the initial, as such demonstrates better properties as a candidate for solar cell applications and in other optoelectronic devices.
\end{abstract}

DOI:10.46481/jnsps.2021.352

Keywords: Perovskite, Photovoltaic solar cell, Optical, Structural

Article History :

Received: 17 August 2021

Received in revised form: 05 October 2021

Accepted for publication: 07 October 2021

Published: 29 November 2021

(C)2021 Journal of the Nigerian Society of Physical Sciences. All rights reserved. Communicated by: E. Etim

\section{Introduction}

Traditionally, silicon has been the most widely used materials in most successful photovoltaic solar cells. However, high processing or manufacturing cost, unavailability of effective mass production technique, reflection, and recombination and absorption losses limit the use of silicon photovoltaic solar cells. Perovskite materials which are generally photonic materials have shown great promising qualities to replace silicon as solar cell materials. The Lead-based halide perovskite

\footnotetext{
${ }^{*}$ Corresponding author tel. no:

Email address: imagaji05@gmail .com (I. Magaji)
}

are considered to be most promising material for solar cell absorbers [1]. However the environmental hazard and the toxicity nature associated with the lead-based halide perovskite still remain a major concern. Recently, there has been tremendous efforts in developing new halide perovskite which will potentially turnaround the instability and toxicity problems associated with emerging lead-based perovskite solar cell devices. Indium (In)-based double perovskite; $\mathrm{Cs}_{2} \mathrm{InBiCl}_{6}$ and $\mathrm{Cs}_{2} \mathrm{InSbCl}_{6}$ were proposed to be good return photovoltaic solar cell material, evidently due to the suitable direct band gaps foretold by SpinOrbit Coupling with Hybrid Functional (SOC+HSE) calculation, minimal effective masses for electron-hole, and thermal stability towards decomposition [5]. The large dielectric con- 
stant [2], low cost fabrication [3], high optical absorption coefficient [4], low exciton binding energy, [5], efficient solar absorption when used as light absorbing layer [6], long carrier diffusion length [7], and swift increase in power conversion efficiency (PCE) [8], are among the properties that makes the double halide and other similar perovskite solar cells very promising for commercialization. The major concern of the proposed indium (In) based double perovskite is the stability of indium (In) state specifically against oxidizing to In (III) [1]. Point defect is a promising way to control such instability particularly against reduction oxidation reaction (REDOX). In semiconductors, point defects play a fundamental role in enhancing material properties. Substitutional point defect was employed by total substitution of indium with gold elements in the compound. Generally, transition metals when used as dopant improves material conductivity. The surface plasmon resonance (SPR) property of gold influences the used of it as the substitute in the compound. In this work, first principle DFT and linear response TDDFT calculations were used to study the effect of Substitutional point defect of transitional metal (gold) alloyed along Indium (In) region of $\mathrm{Cs}_{2} \mathrm{InSbCl}_{6}$ for potential solar cell application.

\section{Computational Details}

The first principle calculations were carried out within the frame work of DFT and TDDFT by the use of plane-wave $(\mathrm{Pw})$ pseudopotential technique as implemented in quantum ESPRESSO suite. Generalized gradient approximation (GGA) was used as exchange-correlation functional formulated by Perdew Burke Ernzerhof (PBE). The lead-free halide perovskite compound $\left(\mathrm{Cs}_{2} \mathrm{InSbCl}_{6}\right)$ is a face-centered-cubic (FCC) which is a member of the cubic lattice structure in the space group Fm-3m. It simple primitive cell contains 10 atoms. However, a super cell of 40 atoms was used throughout the course of this work. The structural parameters (lattice parameters and internal atomic coordinate) were optimized and fully relaxed using Broyden-Flectcher-Goldfarb-Shanno (BFGS) quasi-Newton algorithm. 60 and 240 Ry were set for the convergence test of the kinetic energy cut-off for wave function and charge densities respectively. The Monkhorst-packk-point mesh sampling of Brillouin zone integration was set as $6 \times 6 \times 6$ and a denser k-point mesh of $12 \times 12 \times 12$ for density of state (DOS). $10^{-7}$ was set as the convergence threshold for self-consistent-field (SCF) iteration. The output of the SCF calculation provides the value of pressure $\mathrm{P}$ in equation 1 which was used to calculate the bulk modulus of the materials.

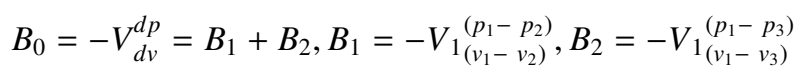

Where $B_{0}$ is the bulk modulus, $\mathrm{V}$ is the volume of the structure, and $\mathrm{P}$ is the pressure

\section{Result and Discussion}

\subsection{Structural properties}

The optimized crystal lattice parameters obtained are $\mathrm{a}=$ $\mathrm{b}=\mathrm{c}=11.342 \AA$ and $\alpha=\beta=\gamma=90^{\circ}$. The value obtained is in consistent with the experimental value $11.32 \AA$ [9]. The cesium Cs atom site (1) in Figure 1(a) was observed to have a bond with at least an atom site of each of the element existing in the material. It has ten (10) neighboring atoms sites with three (3) Indium (In), 3 Chlorine (Cl), two (2) Antimony ( $\mathrm{Sb}$ ), and 2 cesium (Cs) atoms. A total bond length of $30.594 \AA$ exists between the Cs atom site (1) and the neighboring atoms. The alloying of $\mathrm{Au}$ element in the In site of the compound reduces the total bond length to $30.588 \AA$. The alloyed compound $\mathrm{Cs}_{2} \mathrm{AuSbCl}_{6}$ figure 1(b) having lesser total bond length when compared to the initial compound $\mathrm{Cs}_{2} \mathrm{InSbCl}_{6}$ is likely to be more stable. This is because the lesser the bond length the stronger the bond [10].

The strength, hardness, compressibility or flexibility of materials is often determined by the bulk modulus of the material. The bulk modulus (which is a function of pressure and volume) of the material was calculated (using equation 1). The result obtained shows that the bulk modulus of the material $\mathrm{Cs}_{2} \mathrm{InSbCl}_{6}$ calculated is $28.44 \mathrm{Gpa}$. The substitution of In with $\mathrm{Au}$ raises this property to $30.93 \mathrm{Gpa}$. The ability of a material to resist deformation under applied stress is often defined by its bulk modulus [11]. Greater value of the bulk modulus implies strong resistance to deformation and it indicates high compressive strength [12]. This means that the material $\left(\mathrm{Cs}_{2} \mathrm{AuSbCl}_{6}\right)$ will offer more durability and resistance to deformation and as such it is suitable for solar cell application [11].

\subsection{Electronic Properties}

The band structure of the compounds $\mathrm{Cs}_{2} \mathrm{InSbCl}_{6}$ and $\mathrm{Cs}_{2} \mathrm{AuSbCl}_{6}$ were calculated using the projected augmented wave (PAW) within DFT-GGA functionals. The band was calculated in the high symmetry directions of the Brillion zone (X $-\Gamma-\mathrm{W}-\Gamma-\mathrm{L}$ ) for both compounds. It is observed that both materials demonstrate semiconducting behavior. The structures clearly reveals that both the valence band maximum (VBM) and the conduction band minimum (CBM) exist at $\Gamma$-point symmetry. This indicates that the parent compound $\left(\mathrm{Cs}_{2} \mathrm{InSbCl}_{6}\right)$ and the alloyed $\left(\mathrm{Cs}_{2} \mathrm{AuSbCl}_{6}\right)$ are direct band gap materials. The band gap of the material (Figure 2(b)) increased to $1.25 \mathrm{eV}$ after substitution of In with $\mathrm{Au}$ element $\left(\mathrm{Cs}_{2} \mathrm{AuSbCl}_{6}\right)$ in the parent compound (Figure 2(a)) which has a band gap value of 0.99 $\mathrm{eV}$ that is in excellent agreement with the value of $0.98 \mathrm{eV} \mathrm{ob-}$ tained by [1] and most recently $1.02 \mathrm{eV}$ obtained by [9]. The density of state (DOS) shows the number of available states in the system, the number of the allowed electron (hole) states per volume at given energy. Thus, it's a tool for determining the energy distributions and carrier concentrations within semiconductor [13].

The plotted DOS (Figure 3(a) and 3(b)) clearly reveals the semiconducting behavior of the materials. It clearly shows the existence of energy range below and above the Fermi level (0 $\mathrm{eV})$. It shows that the higher peaks are situated at the valence band for $\mathrm{Cs}_{2} \mathrm{InSbCl}_{6}$ (Figure 3(a)) and at conduction band for $\mathrm{Cs}_{2} \mathrm{AuSbCl}_{6}$ (Figure 3(b)). This indicates the presence of many electrons at the particular energy state. 

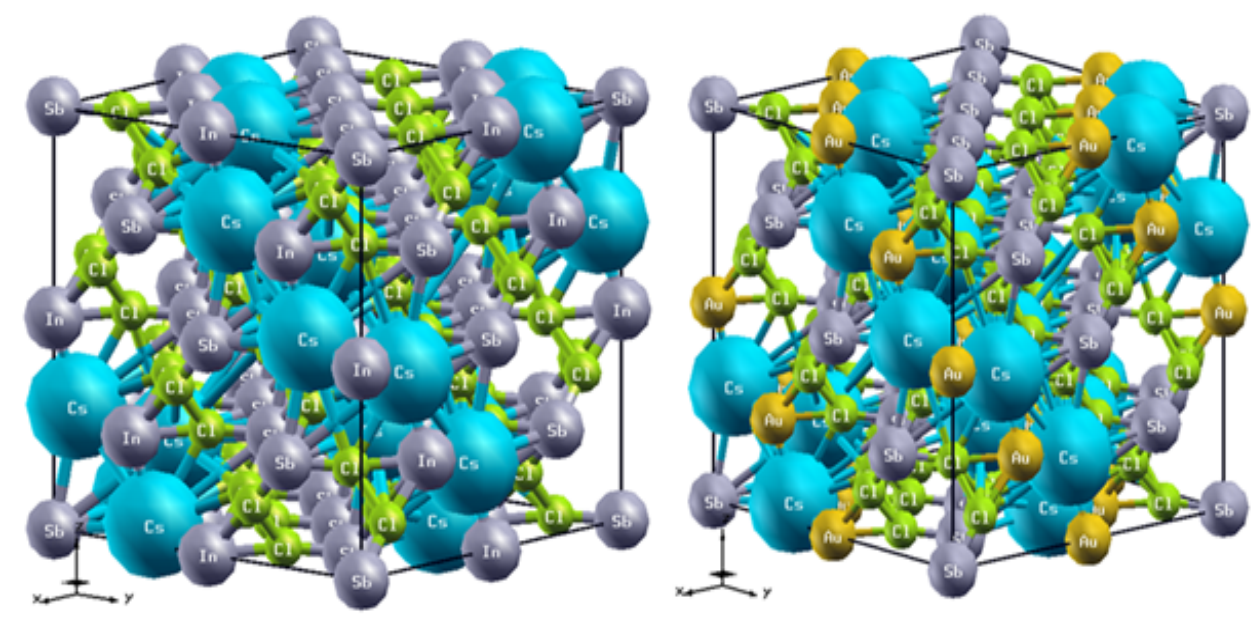

Figure 1. Crystal Structure
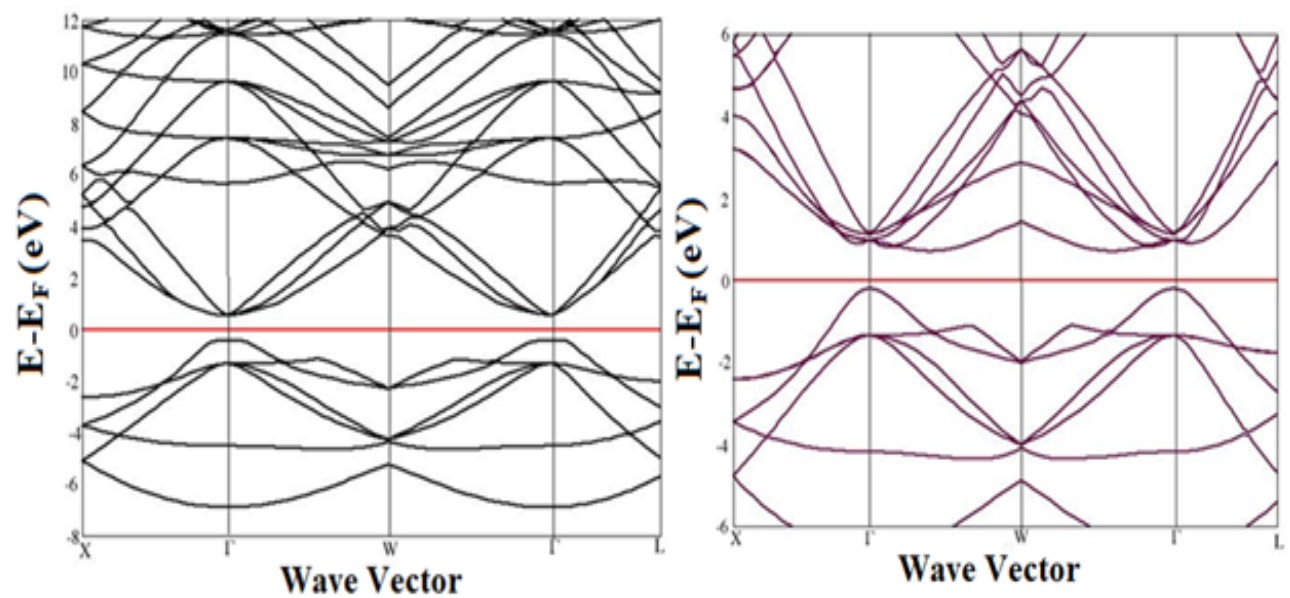

Figure 2. Band structure
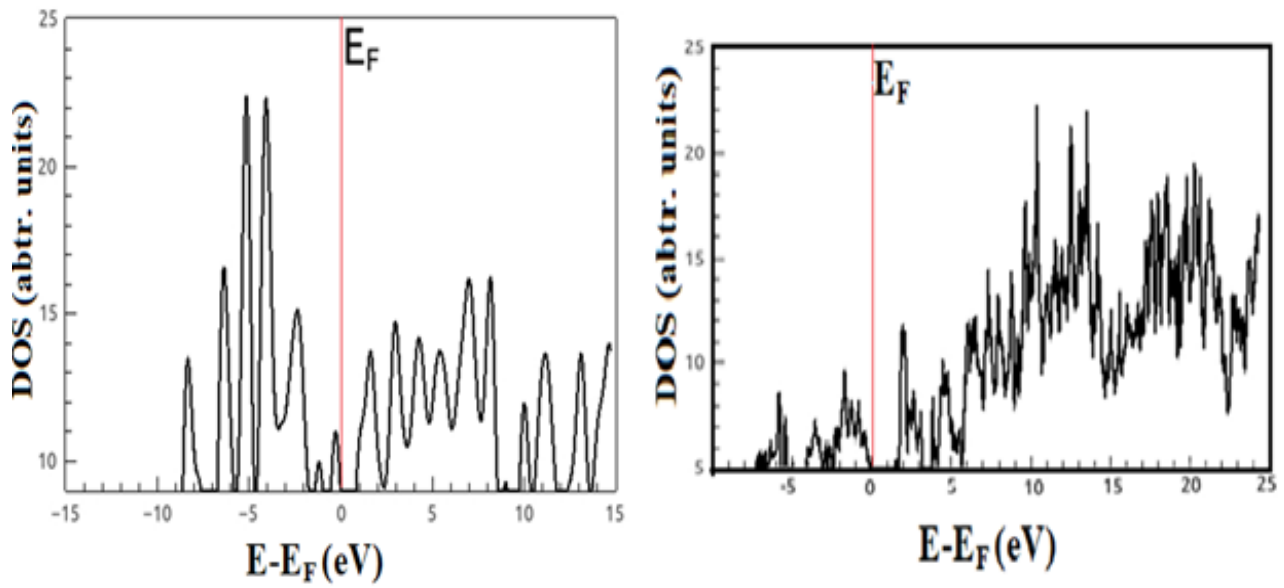

Figure 3. Density of State (DOS)

\subsection{Optical properties}

The study of optical properties involves the determining material response to an applied electromagnetic field particularly to visible light [14].The knowledge is essential in determina- tion of the efficiency of photovoltaic materials. Theoretically, an important function used in solid state physics to determine the linear response is the complex dielectric function [15]. The dielectric function $\varepsilon(\omega)=\varepsilon_{1}(\omega)+i \varepsilon_{2}(\omega)$ (where $\varepsilon_{1}(\omega)$ is 

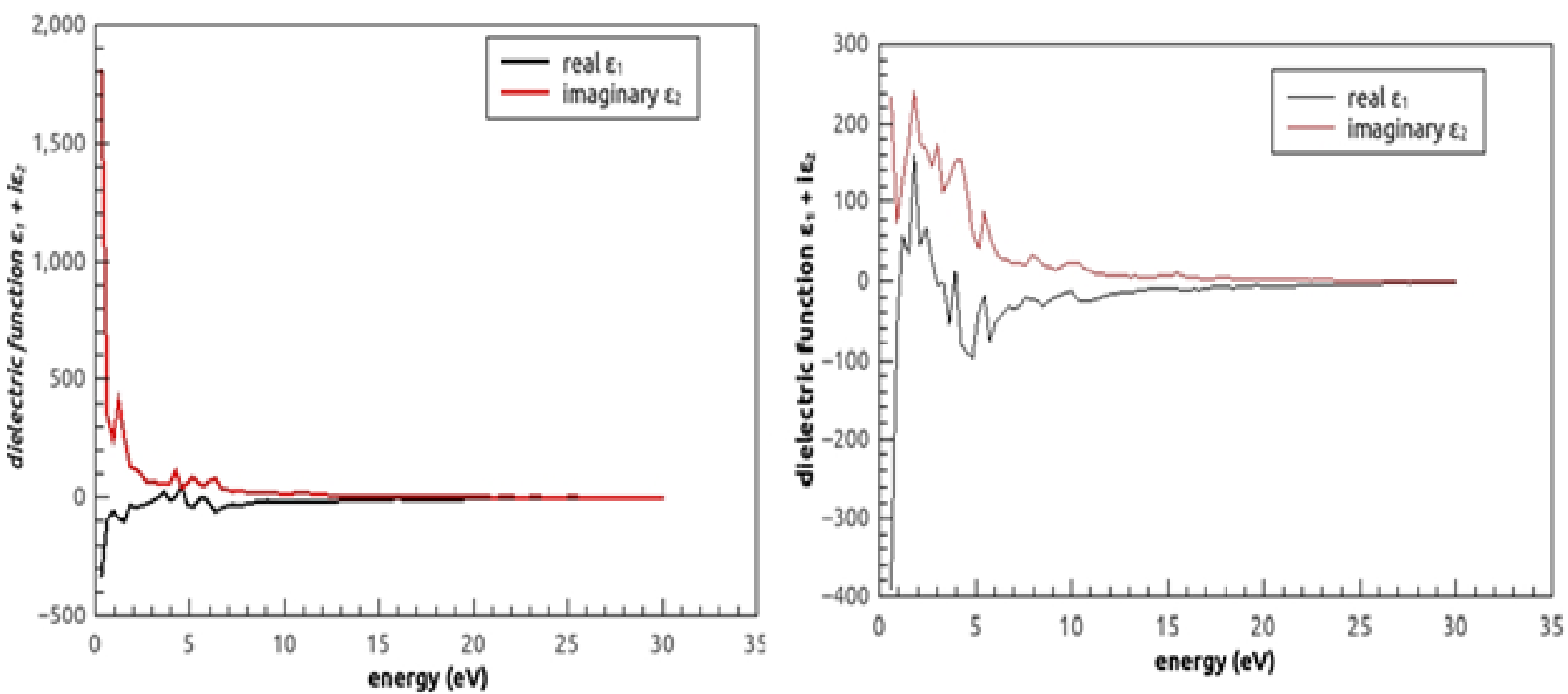

Figure 4. Dielectric Functions

the real part and $i \varepsilon_{2}(\omega)$ is the imaginary part) of the materials were calculated within the linear response TDDFT. The optical transition mechanism is illustrated by the imaginary part of the dielectric function. A greater value for dielectric constant of material indicates that the material has comparatively low charge carrier recombination rate and as such the entire performance of the optoelectronics material will be improved [13]. The result obtained for $\mathrm{Cs}_{2} \mathrm{InSbCl}_{6}$ (Figure 4(a)) shows that the value of the imaginary part $\left(\varepsilon_{2}\right)$ becomes zero at about $23.5 \mathrm{eV}$. This means that the material becomes transparent above 23.5 $\mathrm{eV}$ [14]. Generally, the value of $\varepsilon_{2}$ becomes nonzero immediately absorption starts. It can be seen that for $\varepsilon_{2}$ the main peaks are located at $2 \mathrm{eV}, 3 \mathrm{eV}, 4 \mathrm{eV}$, and $5 \mathrm{eV}$ (Figure 4(a)). An important remark to make from the real part $\left(\varepsilon_{1}\right)$ plot is that $\varepsilon_{1}$ decreases to negative values. The negative values of $\left(\varepsilon_{1}\right)$ indicates that at the particular energy level, there is mostly rejection of the incidence electromagnetic waves from the medium, as such the material exhibit metallic behavior [14]. As such it serves as protective means from the radiations in the energy region. Comparing the result with the alloyed compound $\left(\mathrm{Cs}_{2} \mathrm{AuSbCl}_{6}\right)$ Figure 4(b) shows that the alloyed material will absorb radiations up to about $24 \mathrm{eV}$ which is higher than the initial compound $(13 \mathrm{eV})$. This is in line with the difference in the electronic band gap obtained from the band structure (Figure 2(a) and (b)). Also, it is observed that here's a difference in the high positive peak value of $\varepsilon_{1}$ with about $100 \mathrm{eV}$ that exists between the initial (about $50 \mathrm{eV}$ ) and the alloyed compound (about $150 \mathrm{eV}$ ). This means that the alloyed compound is a good absorber of radiations. As such it is considered as potential good photovoltaic material for optoelectronics application.

The absorption coefficient spectrum provides knowledge on the extent at which material absorbs photons. It indicates how far the light of a specific energy can penetrate into a material before being absorbed [14]. Figure 5(a) shows the absorption coefficient graph which provides information about optimum solar energy conversion efficiency. It reveals the absorption spectra for $\mathrm{Cs}_{2} \mathrm{InSbCl}_{6}$ and $\mathrm{Cs}_{2} \mathrm{AuSbCl}_{6}$. The absorption abruptly rises for energies higher than $0.5 \mathrm{eV}$ which is in line with the band gap energy value. The absorption values are in the order of $10^{4} \mathrm{~cm}^{-1}$ from the infrared to visible and down to the ultraviolet range ( $1.24 \mathrm{eV}$ to $1.7 \mathrm{eV}$ to above $3.3 \mathrm{eV})$. Several peaks were noticed to exist with $\mathrm{Cs}_{2} \mathrm{InSbCl}_{6}$ having its highest peak located just within the infrared energy region while the compound $\mathrm{Cs}_{2} \mathrm{AuSbCl}_{6}$ was noticed to have its utmost peaks located within the ultraviolet region (above $3.3 \mathrm{eV}$ ). This affirms the result obtained by the DFT calculation of the electronic band structure (Figure 2(a) \& (b)) which reveals that the compound $\mathrm{Cs}_{2} \mathrm{AuSbCl}_{6}$ has a wider band gap than $\mathrm{Cs}_{2} \mathrm{InSbCl}_{6}$. This is evidently the reason for the difference in the energy range of the highest peaks. An important remark to make is that the material with higher peak in the high energy region will be a good absorber material and as such it is considered as a better candidate for photovoltaic applications.

The electron energy loss function EELS is a vital optical parameter used in explaining the energy loss of a fast electron traversing a material which is large at plasma frequency [14]. Figure 5(b) represent the corresponding EELS in function of photon energy of the materials. The prominent peaks were found to be at $28.02 \mathrm{eV}$ for $\mathrm{Cs}_{2} \mathrm{InSbCl}_{6}, 29.5 \mathrm{eV}$ for $\mathrm{Cs}_{2} \mathrm{AuSbCl}_{6}$. The sharp maxima peak for EELS indicates the existence of plasma resonance which appears at a specific incident light frequency which corresponds to the trailing edges in the reflection spectra often called plasma frequency $\left(\omega_{\rho}\right)$. The imaginary part of the dielectric function is zero at this point of energy which indicates rapid reduction in reflectance [15].

Reflectivity is an important optical parameter used to describe 

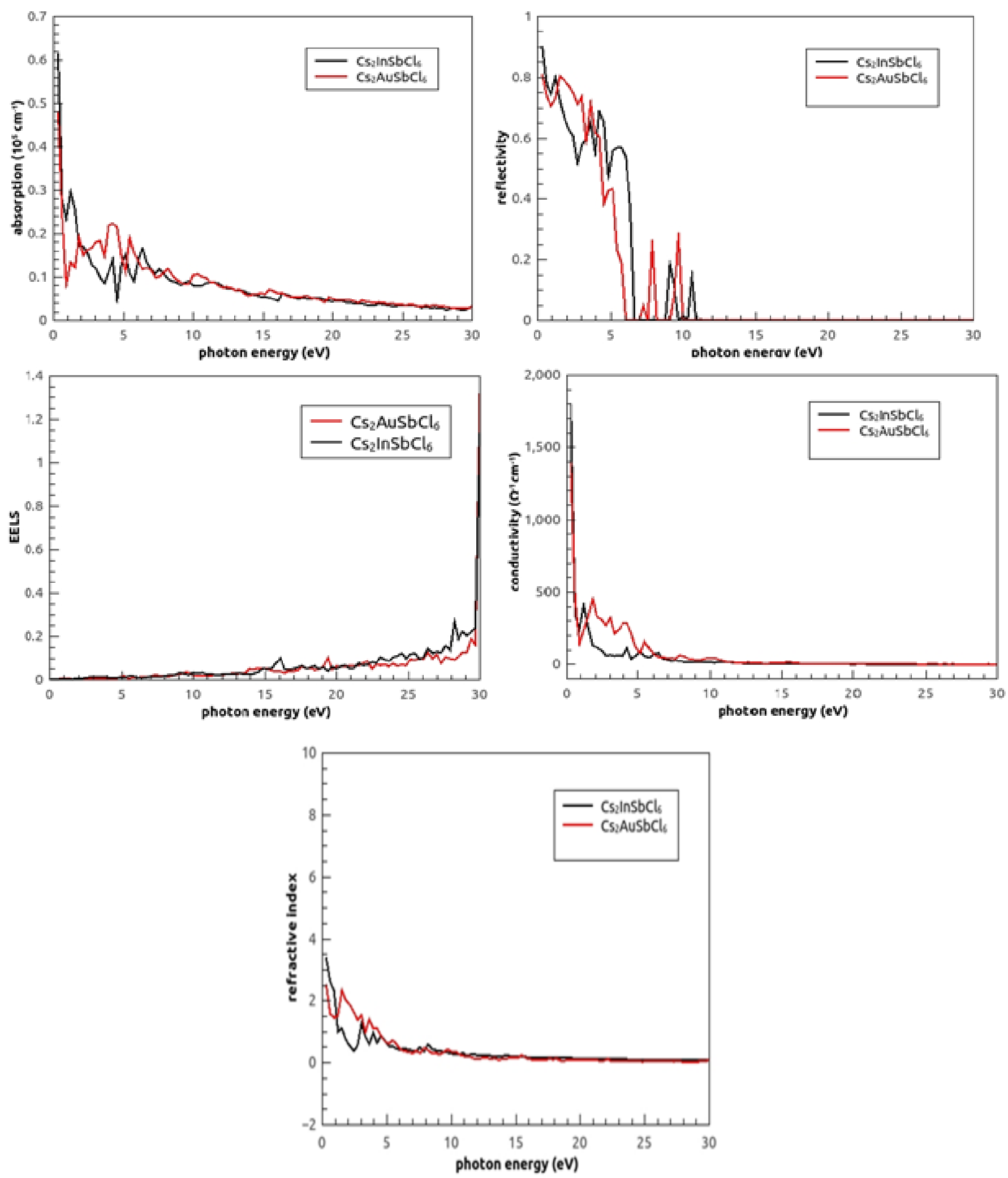

Figure 5. (a) Absorption Coefficient, (b) Electron Energy Loss Spectra, (c) Reflectivity, (d) Conductivity, (e) Refractive Index

the ratio of reflected photon energy from material surface to the photon energy incident on the surface. Figure 5(c) shows the reflectivity of the materials $\left(\mathrm{Cs}_{2} \mathrm{InSbCl}_{6}\right.$ and $\left.\mathrm{Cs}_{2} \mathrm{AuSbCl}_{6}\right)$ as function of photon energy. The first edges were found to be at $90 \%$, and $80 \%$ for $\mathrm{Cs}_{2} \mathrm{InSbCl}_{6}$, and $\mathrm{Cs}_{2} \mathrm{AuSbCl}_{6}$ respectively. These edges were noticed to be within the low energy level. 
The reflectivity begins to decrease as the energy increases from infrared region. It then increases to other peaks of 75, and 70 percent for $\mathrm{Cs}_{2} \mathrm{AuSbCl}_{6}$ and $\mathrm{Cs}_{2} \mathrm{InSbCl}_{6}$ respectively. These peaks were found to be located from visible to ultraviolet energy range. The decrease in the reflectivity in the high energy region means that the material is transmitting in the ultraviolet wavelength as a result of lower reflectance within the energy range. An important remark to make is that, the material with high reflectivity in the high energy region can be used as a good coating material to avoid solar heating [14].

Conductivity is an important optical property that provides information on the electrical conductivity of material. The measure of ease at which heat or electric charge pass through a material is termed conductivity. Figure 5(d) below shows the conductivity of the materials. It is observed that the material $\mathrm{Cs}_{2} \mathrm{InSbCl}_{6}$ has its own highest optical conductivity value of $400 \Omega^{-1} \mathrm{~cm}^{-1}$. The conductivity rises to about 500 $\Omega^{-1} \mathrm{~cm}^{-1}$ for $\mathrm{Cs}_{2} \mathrm{AuSbCl}_{6}$. The increase in the photoconductivity of $\mathrm{Cs}_{2} \mathrm{AuSbCl}_{6}$ is due to high absorption of photons [14]. The material $\mathrm{Cs}_{2} \mathrm{AuSbCl}_{6}$ will consequently be a better candidate for photovoltaic applications.

The refractive index of a material is a dimensionless quantity which describes how much light travels or refracted after entering a material [15]. Figure 5(e) shows the refractive index $n(\omega)$ as a function of photon energy for the materials. The static refractive index $n(0)$ was found to be 2.5 and 3.4 for $\mathrm{Cs}_{2} \mathrm{AuSbCl}_{6}$ and $\mathrm{Cs}_{2} \mathrm{InSbCl}_{6}$ respectively. From the graph, the materials possess a high refractive index at infrared region around (1.24 $\mathrm{eV}-1.7 \mathrm{eV})$ to visible region $(1.7 \mathrm{eV}-3.3 \mathrm{eV})$ and drastically decrease at higher energy from the visible to ultraviolet region (above $3.3 \mathrm{eV}$ ). Furthermore, after $2.5 \mathrm{eV}$, and $6.0 \mathrm{eV}$ the velocity of light is greater than the light celerity because $\mathrm{n}(\omega)$ is less than one for $\mathrm{Cs}_{2} \mathrm{InSbCl}_{6}$, and $\mathrm{Cs}_{2} \mathrm{AuSbCl}_{6}$ respectively. The $\mathrm{n}(0)$ attain by $\mathrm{Cs}_{2} \mathrm{AuSbCl}_{6}$ (2.5) which is lower than the initial $\mathrm{Cs}_{2} \mathrm{InSbCl}_{6}$ (3.4) will be less dense as such, the light rays will bend more away from the normal line. The result obtained for $\mathrm{Cs}_{2} \mathrm{AuSbCl}_{6}$ (2.5) is in consistent with the result obtained for other lead-free halide $\mathrm{Cs}_{2} \mathrm{TiI}_{6}$ double perovskite of $\mathrm{n}(0)=$ 2.6 [18]. As such, $\mathrm{Cs}_{2} \mathrm{AuSbCl}_{6}$ can be considered as a good photovoltaic material.

\section{Conclusion}

In this research work, investigation was carried out on the effect of substitutional point defect in the structural, electronic and optical properties of a lead-free halide perovskites $\mathrm{Cs}_{2} \mathrm{InSbCl}_{6}$ for photovoltaic application within the framework of DFT-GGA (for the structural and electronic properties) and TDDFT (for optical properties) as implemented in quantum ESPRESSO (q.e-6.5).

A substitutional point defect was performed in the indium site of the compound with substitution of indium with a gold element. The effect was investigated on both the structural, electronic and optical properties. The result of the structural investigation shows that the alloyed compound $\left(\mathrm{Cs}_{2} \mathrm{AuSbCl}_{6}\right)$ has less total bond length when compared with the initial compound $\left(\mathrm{Cs}_{2} \mathrm{InSbCl}_{6}\right)$ and such it makes more stable. The higher magnitude of bulk modulus attained by the alloyed compound means that it will offer more durability and resistance to deformation which makes it suitable for solar cell application. The electronic band structure (Figure 2) shows the substitution widened the band gap from $0.99 \mathrm{eV}$ to $1.25 \mathrm{eV}$. This increase in the band gap value of the defect compound indicates higher absorption of photons energy. The result of the optical properties shows that the defect material has higher absorption coefficient, lower reflectivity and higher optical conductivity when compared with the initial, this means that the defect compound is a good photovoltaic material and potentially better candidate for solar cell application.

\section{References}

[1] Z. Xiao, K. Du, W. Meng, J. Wang, D. B. Mitzi \& Y. Yan, "Intrinsic instability of Cs2In (I) M (III) X6 (M= Bi, Sb; X= halogen) double perovskites: a combined density functional theory and experimental study." Journal of the American Chemical Society 139 (2017) 6054

[2] T. Baikie, Y. Fang, J. M. Kadro, M. Schreyer, F. Wei, S. G. Mhaisalkar, M. Graetzel \& T. J. White, "Synthesis and crystal chemistry of the hybrid perovskite (CH 3 NH 3) PbI 3 for solid-state sensitised solar cell applications." Journal of Materials Chemistry A 1 (2013) 5628.

[3] H. Yu, F. Wang, F. Xie, W. Li, J. Chen \& N. Zhao, "The role of chlorine in the formation process of "CH3NH3PbI3-xClx" perovskite." Advanced Functional Materials 24 (2014) 7102.

[4] H. Yu, H. Lu, F. Xie, S. Zhou \& N. Zhao. "Native defect-induced hysteresis behavior in organolead iodide perovskite solar cells." Advanced Functional Materials 26 (2016) 1411.

[5] W. Zhao, Z. Yao, F. Yu, D. Yang \& Shengzhong Liu, "Alkali metal doping for improved $\mathrm{CH} 3 \mathrm{NH} 3 \mathrm{PbI} 3$ perovskite solar cells." Advanced science $\mathbf{5}$ (2018) 1700131.

[6] C. C. Stoumpos, C. D. Malliakas \& M. G. Kanatzidis, "Semiconducting tin and lead iodide perovskites with organic cations: phase transitions, high mobilities, and near-infrared photoluminescent properties." Inorganic chemistry 52 (2013) 9019.

[7] J. Shi, S. Yun, "First principle DFT calculations for perovskite solar cells". Wiley-VCH \& Co. 19 (2018) 487.

[8] H. Zhou, Q. Chen, G. Li, S. Luo, T. Song, H. Duan, Z. Hong, J. You, Y. Liu \& Yang Yang, "Interface engineering of highly efficient perovskite solar cells." Science 345 (2014) 542.

[9] Y. Li, First-Principles Study of Hybrid Halide Perovskites and Beyond for Optoelectronic Applications. University of California, San Diego, 2020.

[10] R. C. King, Introduction to microelectronics theory. Georgia institute of technology, 2005.

[11] M. Houari, B. Bouadjemi, A. Abbad, W. Benstaali, S. Haid, T. Lantri, A. Zitouni, S. Bentata, B. Bouhafs, and Z. Aziz. "Structural, electronic and optical properties of cubic fluoroelpasolite Cs2NaYF6 by density functional theory." Chinese Journal of Physics 56 (2018) 1756.

[12] A. Natik, Y. Abid, R. Moubah, F. Khelfaoui, E. K. Hlil, H. Zaari, A. Benyoussef, M. Abid \& H. Lassri, "Structural, elastic, electronic and optical properties of $\mathrm{RbPbI} 3$ perovskites studied using ab-initio calculations." Phase Transitions 93 (2020) 54.

[13] J. Islam \& A. A. Hossain, "Narrowing band gap and enhanced visiblelight absorption of metal-doped non-toxic $\mathrm{CsSnCl} 3$ metal halides for potential optoelectronic applications." RSC Advances 10 (2020) 7817.

[14] A. Rahman, Z. Rahaman \& A. Rahman, "The structural, elastic, electronic and optical properties of $\mathrm{MgCu}$ under pressure: A first-principles study.” International Journal of Modern Physics B 30 (2016) 1650199.

[15] A. Lawal, A. Shaari, R. Ahmed \& N. Jarkoni, "First-principles investigations of electron-hole inclusion effects on optoelectronic properties of Bi2Te3, a topological insulator for broadband photodetector.' Physica B: Condensed Matter 520 (2017) 69. 